\section{OC 8491 PREPVACC: A PHASE III, MAMS ADAPTIVE PROPHYLACTIC HIV VACCINE TRIAL WITH A SECOND RANDOMISATION TO COMPARE F/TAF WITH TDF/FTC PREP}

\begin{abstract}
${ }^{1}$ Sarah Joseph*, ${ }^{2}$ Pontiano Kaleebu, ${ }^{2}$ Eugene Ruzagira, ${ }^{2}$ Christian Holm Hansen, ${ }^{2}$ Janet Seeley, ${ }^{2}$ Vincent Basajja, ${ }^{1} J o n a t h a n$ Weber, ${ }^{3}$ Julie Fox, ${ }^{1}$ Cherry Kingsley, ${ }^{4}$ Said Aboud,

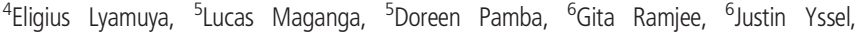
${ }^{7}$ Edna Viegas, ${ }^{7}$ llesh Jani, ${ }^{8}$ Fran Priddy, ${ }^{9}$ Charlotta Nilsson, ${ }^{10}$ Arne Kroidl, ${ }^{11}$ Giuseppe Pantaleo, ${ }^{12}$ Merlin Robb, ${ }^{13}$ Angela Crook, ${ }^{13}$ David Dunne, ${ }^{13}$ Sheena Mccormack. ${ }^{1}$ Imperial College London, UK; ${ }^{2}$ MRC/UVRI and LSHTM Uganda Research Unit; ${ }^{3}$ King's College London; ${ }^{4}$ Muhimbili University of Health and Allied Sciences, Tanzania; ${ }^{5}$ NIMR-Mbeya Medical Research Center; ${ }^{6}$ South African Medical Research Council, Cape Town, South Africa; 'Instituto Nacional de Saúde, Maputo, Mozambique; ${ }^{8}$ International AIDS Vaccine Initiative, New York, USA; ${ }^{9}$ Public Health Agency of Sweden; Stockholm, Sweden; ${ }^{10}$ Ludwig-Maximilians-Universität, Munich, Germany; ${ }^{11}$ Centre Hospitalier Universitaire Vaudois, Switzerland; ${ }^{12}$ US Military HIV Research Programme, Washington DC, USA; ${ }^{13}$ MRC Clinical Trials Unit, London, UK
\end{abstract}

\subsection{6/bmjgh-2019-EDC.23}

Background There remains an urgent need for a prophylactic HIV vaccine to control generalised epidemics. PrEP has demonstrated effectiveness of $86 \%$ and is recommended by WHO; uptake is generally high, but retention is disappointing in some settings. The EDCTP2 project PrEPVacc will assess the efficacy of two combination prophylactic vaccine regimens (DNA, MVA and Env protein/adjuvant) each compared to placebo and the proportion of infections averted by F/TAF in comparison to TDF/FTC. A Registration Cohort, recruiting HIV negative volunteers at risk of HIV will precede the trial. Methods The PrEPVacc partnership agreed that 70\% vaccine efficacy had public health relevance. The trial uses nstage software for multi-arm, multi-stage designs (MAMS) and the averted infections ratio (AIR) methodology with participants randomised (i) $1: 1: 1$ to active product or placebo (ii) $1: 1$ to TDF/FTC : F/TAF until week 26 (presumed peak immunogenicity). Access to PrEP in the Registration Cohort and after week 26 will be standard of care. HIV seroconversions occurring between weeks 0-26 will inform the PrEP analysis, incorporating HIV incidence amongst those who do not take up PrEP locally in the Registration Cohort. Seroconversions after week 26 will inform vaccine analyses.

Results Up to 556 participants per group affords 92\% power to detect vaccine efficacy of $70 \%$ at the final analysis, assuming incidence of 4/100-person years and 10\% loss with $81 \%$ and $97 \%$ power to conclude that $\mathrm{F} / \mathrm{TAF}$ can avert half or more of the infections prevented by TDF/FTC if effectiveness of TDF/FTC is $70 \%$ and $80 \%$, respectively.

Conclusion PrEPVacc adopts a pragmatic approach to uncertainties around HIV incidence in settings where PrEP is increasingly available. This innovative adaptive trial design uses validated software to determine vaccine efficacy and a novel methodology to evaluate a new PrEP agent, overcoming the challenge of demonstrating non-inferiority when adherence to TDF/FTC is high and the number of outcome events very low.

\section{OC 8494 DRIVING REDUCED AIDS-ASSOCIATED MENINGO-ENCEPHALITIS MORTALITY}

\footnotetext{
${ }^{1}$ Angela Loyse*, ${ }^{2,4}$ Godfrey S Mfinanga, ${ }^{3}$ Cecilia Kanyama, ${ }^{6}$ Charles Kounfack, ${ }^{4}$ Sokoine Lesikari, ${ }^{5}$ Chimwemwe Chawinga, ${ }^{1}$ Muirgen Stack, ${ }^{1}$ Sile Molloy. ${ }^{1}$ DREAMM, St Georges, University of London, UK; ${ }^{2}$ DREAMM, NIMR; ${ }^{3}$ DREAMM, UNC Project-Malawi, Kamuzu Central Hospital, Malawi; ${ }^{4}$ NIMR, Muhimbili Medical Research Centre, Dar es Salaam, Tanzania; ${ }^{5}$ UNC Project-Malawi, Kamuzu Central Hospital, Malawi; ${ }^{6}$ Hōpital Central Yaoundé/Site ANRS, Cameroon
}

10.1136/bmjgh-2019-EDC.24
Background DREAMM is an implementation study aiming to reduce meningo-encephalitis related mortality. Delays in diagnosis and treatment through poor access to diagnostics and treatments are significant contributing factors to the ongoing high mortality of HIV-associated central nervous system (CNS) infections, causing up to $25 \%$ of all HIV-related deaths in sub-Saharan Africa.

Methods A before-after design is being implemented across 3 sites in Africa; Lilongwe, Malawi, Dar Es Salaam, Tanzania and Yaoundé, Cameroon. The study is composed of 3 phases: Observation, Training and Implementation.

Results The observation phase (audit of practice) happened between November 2016 - May 2017 in Malawi and Tanzania. Overall, 110 patients were included. Ten-week all-cause mortality was 64\% (42/66) in Tanzania and $37 \%(13 / 35)$ in Malawi. Approximately $75 \%$ of patients were ART experienced. Across sites, $76.6 \%$ of patients presented with abnormal mental status, with a median baseline CD4 count of 50 cells $/ \mu$ L. Only $2 / 75$ patients in Tanzania had a lumbar puncture ordered compared to $27 / 35$ in Malawi. All patients in Tanzania received empirical Fluconazole monotherapy whereas almost $1 / 3$ patients in Malawi (11/35) were treated with Amphotericin B which is not readily available in both countries.

The training phase (completed in November 2017 for Malawi and Tanzania) used the train-the-trainer approach. Interactive workshops on using rapid diagnostic tests (RDTs), performing basic microbiological techniques and safe administration of medicines were chosen as the most pertinent to reducing mortality. Patient and laboratory pathways were optimised afterwards by increasing the routine laboratory capacity, performing CSF analysis, providing infectious diseases mentorship for clinicians and procuring RDTs and reagents not locally available.

Implementation is underway in Malawi and Tanzania and the audit phase is scheduled for autumn in Cameroon. Upon completion, the project is expected to create a sustainable approach to reduce the high mortality of HIV-related meningo-encephalitis with the optimised patient and laboratory pathways embedded within routine care.

\section{OC 8499 THE T-CELL VACCINE STRATEGY: GLOBALLY RELEVANT AIDS VACCINE EUROPE-AFRICA TRIALS PARTNERSHIP (GREAT)}

${ }^{1}$ Tomáš Hanke*, ${ }^{2}$ Patricia Fast, ${ }^{3}$ Pontiano Kaleebu, ${ }^{4}$ Walter Jaoko, ${ }^{5}$ Eduard Sanders, ${ }^{6}$ William Kilembe, ${ }^{2}$ Hester Kuipers, ${ }^{7}$ Brenda Okech, ${ }^{8}$ Jill Gilmour. ${ }^{1}$ University of Oxford, UK; ${ }^{2}$ International AIDS Vaccine Initiative (IAVI), New York, USA; ${ }^{3}$ Uganda Virus Research Institute (UVRI), Entebbe, Uganda; ${ }^{4}$ IAVI- Institute of Clinical Research, Nairobi, Kenya; ${ }^{5}$ Kenya Medical Research Institute (KEMRI)-Wellcome Trust Research Programme, Kilifi and Mtwapa, Kenya; ${ }^{6}$ Zambia-Emory HIV Research Project (ZEHRP), Lusaka, Zambia; ${ }^{7}$ UVRI-IAVI, Entebbe, Uganda; ${ }^{8}$ IAVI Human Immunology Laboratory (HIL), London, UK

\subsection{6/bmjgh-2019-EDC.25}

Background Remarkable progress has been achieved in decreasing AIDS-related deaths and HIV-1 transmission through ART. Nevertheless, an affordable, effective and durable HIV-1 vaccine protection remains the best solution for halting the AIDS epidemic.

Our aim is to develop a vaccine inducing cytotoxic T lymphocytes (CTL), which effectively inhibit HIV-1 replication and complement bnAbs for prevention; such $\mathrm{T}$ cells are likely 